\title{
Exploring The Use of Digital Games for Teaching and Learning
}

\author{
Wan Mohd Nazmee Wan Zainon, Abdullah Zawawi Talib², \\ Mohd Azam Osman ${ }^{3}$
}

School of Computer Sciences, Universiti Sains Malaysia, 11800 USM, Penang, Malaysia

\begin{abstract}
Whether we like it or not, digital gaming and social networking are already widespread activities in our culture. More and more homes are now equipped with video-game consoles, computers, tablets or smart phones. The main purpose of this paper is to present an outcome of a preliminary studies done in exploring the current use of digital games in teaching and learning. This article will discuss how digital games can be used in our education systems and the main benefits of venturing into this research area. Initial investigation suggests that this proposed research will be able to answer those questions about learning games and may help plot an educational design model or guidelines for people and organizations who are interested in developing or fostering the development of digital games for learning.
\end{abstract}

Keywords: Digital Games, Teaching and Learning, Games in Education.

\section{Introduction}

Nowadays, digital games are entertainment software used by a lot of individuals regardless of age, culture or country [1]. In modern day societies, playing with digital games, joining digital game forum or connecting to social networks with embedded games are ordinary activities of daily life. Every day, children are spending countless hours immersed in popular technologies, such as Facebook or Twitter, World of Warcraft, or Sim City, which at first glance may seem like a waste of time. But these genres of technologies like Social Networking, Digital Gaming, and Simulations deserve a deeper look at what is actually occurring [2].

The notion of using digital games and social networking for learning causes some to scratch their head, others to jump for joy, and many to ask questions about this unfamiliar learning medium. These questions often come from people and organizations that are considering delving into the world of learning games but don't know if this is advisable or don't know where to start. 


\section{Research Background}

Playing computer games can be competitive, co-operative or individualistic [3]. The content and contexts embodied in games tend to reflect the interests, fantasies and aspirations of their majority user group. For example, many games involve various aspects of action adventure in which a variety of more or less humanoid opponents can be violently overcome. Others are to do with excelling in sport, completing dangerous missions to retrieve or collect things, or taking on the persona of a warrior or hero and employing a strategy to win.

Digital games use technology to represent reality or to embody fantasy. They provide an environment in which action can be practised or rehearsed with, ultimately, little consequence [4]. Games are played to win or to achieve a goal. It is the playing of the game that is entertaining, with the end result satisfying the majority of players only if challenges have been encountered and difficulties conquered. Table 1 identifies several features of games that can contribute to the player engagement with the software.

Table 1: Aspects of games [5]

\begin{tabular}{|lll|}
\hline Technological & Narrative & Personal \\
\hline \hline Graphics & Novelty & Logic \\
Sound & Story line & Memory \\
& Curiosity & Reflexes \\
& Complexity & Mathematical \\
& & skills \\
& Fantasy & Challenge \\
& & Problem solving \\
& & Visualisation \\
& &
\end{tabular}

Some of these aspects might be usefully incorporated into educational software: visualisation, for example, is a key cognitive strategy, and problem solving skills are critical for the development of independent and resourceful young people [5].
What is captivating for players about games tends to be their structure rather than their content. Structure involves dynamic visuals, interaction, and the presence of a goal and rules that govern play [6]. The key to motivation is winning while remaining challenged. Game playing may be thought of as intrinsically motivating; it is engaged in for its own sake and not to receive external rewards such as money. An important aspect of the 'control' offered by games is that while the computer initiates some actions, the user initiates others. It is this adaptive interactivity that makes games attractive [7].

\section{Digital Games in Education}

Those who believe in using games in education usually start from a common set of assumptions. They observe that game players regularly exhibit persistence, risktaking, attention to detail and problem solving skills, which are all behaviours that ideally would be regularly demonstrated in school. They also understand that game environments enable players to construct understanding actively, and at individual paces, and that well-designed games enable players to advance on different paths at different rates in response to each player's interests and abilities, while also fostering collaboration and just-in-time learning [9].

Advocates for game-based learning tend to adopt one of two very different approaches to designing games for formal education. One group sees the skills students develop while playing games as essential to a twenty-first-century education, and conversely see little progress happening in schools still shackled to a nineteenth-century factory model [10]. They focus on the habits of mind and dispositions needed to collaborate, innovate, problem-solve and communicate effectively in a knowledge-based economy. 
They observe with some accuracy that these skills can all be improved from engagement with commercial, off-the-shelf (COTS) games, or through social networking, blogging, and other forms of user-generated content that fall under the larger banner of participatory culture. They focus on these skills often to the exclusion of traditional academic subject matter, and at least insofar as game-based learning is concerned, they assume the institution of school is highly resistant to reform and find alternate venues and opportunities to foster learning. They imagine the important learning will take place outside of school, and question what value school adds to the process.

Clearly, many COTS games provide opportunities for learning. Examples vary from simulations such as Roller Coaster Tycoon or Civilization that require planning, quantitative skills and significant analysis to Massively Multiplayer Online Role Playing Games (MMORPGs) like World of Warcraft (WoW) or Eve Online, which promote communication, collaboration, and problem-solving skills.

In contrast, the second group tends to concentrate only on applying games in traditional school settings with traditional methods and outcomes. They may pay lip service to 21 st century skills, but they look at the learning that occurs in COTS games and ask, "Why can't we use the form of these successful games to instead foster learning in more traditional academic areas?" In order to integrate games into the existing school environment, they must address several common concerns of teachers [12]:

- Their need to cover mandated content areas;

- Healthy scepticism of new technologies (combined with a lack of infrastructure for these technologies);

- An unfamiliarity with games, and no easy route to game competence.
In addition, proponents of games in school also have to overcome the objections of those parents, teachers and administrators who see games as insufficiently serious, that they are "just play." The solution to all these difficulties tend to be games that can be played in very short bursts of class time, games whose simplicity make them easy to grasp immediately, and games that are stuffed with, what can be recognized as, factual content-often referred to as Integrated Learning Systems (ILS)[12]. These games are often curricular, attempting to teach subject matter that is otherwise advanced through textbooks, lectures, or problem sets (for example Alga-Blaster, Knowledge Munchers, and Word Island). Sadly, while they may go by the name "games", they usually end up bearing little resemblance to the games mentioned above that promote learning of 21 st century skills, instead being little more than interactive quizzes. Resemblance to a game is meaningless when the activity is nothing more than answering multiplechoice questions and when success is measured solely as the percentage of correct answers given expressed as a "score" and presented with a fun animation.

If the first group embraces games and abandons school, this second group often embraces school to the detriment of anything that looks like real gaming.

In spite of their striking differences, we've pointedly avoided suggesting that these impulses - to promote new modes of learning on the one hand, and to adapt to the classroom on the other-are mutually exclusive. One might advocate that games can both build twenty-first-century skills and channel those skills in traditional academic fields. One could also argue that just because such games might be in the service of school, they don't necessarily have to be designed to blend into outmoded forms of schooling. It is unfortunate that when talk turns to how 
games should be implemented in education, the models proposed tend to land heavily at one end of the spectrum or the other.

To succeed, we must look at where the strengths and challenges of both classrooms and games lie and situate "learning games" at the most productive intersection of these separate environments.

\section{Research Objectives}

This study has the following objectives:

\subsection{To investigate how students and children interact with computers}

As certain studies of child-computer interaction suggest, many children actually personify computational objects and often consider computers as being "alive", able to "think", and having a personality. These findings provide an interesting new way of looking at human-computer interaction.

\section{What needs to be investigated?}

This part identifies some areas for consideration - issues that we will be focusing when designing our educational model for digital games:

- Games for more than one player; multiuser interfaces and shared spaces to facilitate collaboration, either synchronous (interactive) or asynchronous.

- The development and use of the facility for asynchronous play of games (such as email chess).

- The anonymity of players, and advantages and disadvantages of this over networks.

- The relationship between people's cognitive, practical and social skills and their ability to play games.

- The effects of stereotyping and violence on the user.

- Motivating disengaged students.
- The effects of, or reasons for, game playing.

- Developing language, problem solving, or mathematical skills.

- An analysis of what learners perceive as goals and how they go about achieving them.

- Gender factors in playing digital games.

\subsection{To assess the current model and the use of (educational) computer games}

This involves looking at educational content and motivational elements in order to come up with suitable representation of concepts and interfaces in designing educational games.

\subsection{To make recommendation on strategies and materials to inte- grate game-like computer activi- ties with other forms of classroom learning}

We consider different learning styles and classroom activities and incorporate elements such as graphics, sound effects, stories, characters, humour, rewards and navigation to make learning a more enjoyable experience.

Guidelines for the design of computer games for use in educational settings should be no different from those for any educational software. Software should incorporate a sound educational philosophy, and should have clearly stated educational objectives and content. Design should include feedback from teachers, students and psychologist. Software should be designed to be inclusive of a wide range of aptitudes and abilities, providing support for the learner and some guidance for teachers planning to use the software. 


\section{Research Methodology}

In order to achieve the research objectives as stated above, this research will be conducted through the following step:

I. Preliminary Study - In this step we will firstly explores various digital games design, concepts, philosophy and culture with the focus especially to the ones that was developed for educational purposes.

II. Propose and Develop New Design Model/ Framework - Based on the identified design, concepts, philosophy and culture found in Step 1, a new digital games design model will be designed and develop.

III. Exploring the Impact of Design in Educational Games - Once a new design model has been developed, proper study will be conducted to help the games developers in understanding how the children (especially students) interact with computers when playing the games.

IV. Designing and Develop Digital Games - An interactive digital games and tools will be design based on the new design model (Step 2) and the study on the impact of the design in educational games (Step 3).

V. Analysing/Evaluating the Proposed Model and Application- The effectiveness of the newly model and applications will be analyzed and evaluated by conducting a descriptive survey methods. Evaluation is one the key points in any research process. It helps to summarize how well the model work compare with current practices.

The collected results of the newly proposed model and applications will be published in different types of publications according to the impact and contribution of the findings.

\section{Discussion \& Future Work}

As games move from being solely a technological tools to spawning a pervasive culture of play, the findings from this research may help to unlock generations of students who are curious, confident and collaborators.

Without a doubt, without using these recent technologies such as digital games, Social networking, Web 2.0, and so on. in the classroom, proper lessons can still be achieved. The main question here is to ask ourselves; Where are we headed with the use digital games, simulations, and social networking technologies in educational practice?

While we cannot say for certain, we can say that the capacity for digital games, simulations and social networking technologies to facilitate and leverage interactive learning is evident enough to warrant further exploration and the development of new best practices. But one does not need to wait for the distant future to understand if and how to implement these technologies. Students today are using these technologies now.

The later stage of research will not only involved researchers in computer sciences field but will also include people from education, professional game developers, teachers and hopefully engage hundreds or thousands of children through direct or indirect participation. The final outcomes of this collaboration will include a wide range of research studies as well as an innovative prototype games that can demonstrate that games can be effective in increasing both motivation and achievement in teaching and learning.

\section{References}

[1] Chow,A.F., Woodford, K. C. \& Maes, J. (2010). Deal or No Deal: using games to improve student learn- 
ing, retention and decision-making. International Journal of Mathematical Education in Science and Technology,pp.1-6.

[2] Facer, K, Joiner R., Stanton, D., Reid, J. Hull, R \& Kirk, D. (2004). Savannah: Mobile gaming and learning. Journal of Computer Assisted Learning (20), pp. 339-409.

[3] Nilsson, M.E. \& Jakobsson, A. (2011). Simulated sustainable societies: Students' reflections on creating future cities in computer games. Journal of Science Education Technology, pp. 33-50

[4] O'Brien, D. (2010).Gaming and simulations: Concepts, methodologies, tools and Applications. Illinois: Information Resources Management Association.

[5] Papastergiou, M. (2009). Digital game-based learning in high school computer science education: Impact on educational effectiveness and student motivation. Computers \& Education, 52(1), pp.1- 12

[6] Wang, Li-Chun \& Chen, MingPuu(2010). The effects of game strategy and preference-matching on flow experience and programming performance in game-based learning. Innovations in Education and Teaching International, 47(1), pp. 39-52.

[7] Schwabe, G., Goth, C. (2005). Mobile learning with a mobile game: design and motivational effects. Journal of Computer Assisted Learning, 21(3), pp. 204-216.

[8] Zyda, M., Thukral, D., Jakatdar, S., Engelsma, J., Ferrans, J., Hans, M., Shi, L., Kitson, F., Vasudevan, V.(2007).Educating the next generation of mobile game developers. IEEE Computer Graphics and Applications, 27(2).

[9] Becker, K. (2007). Digital gamebased learning once removed: Teaching teachers. British Journal of Edu- cational Technology, 38(3), pp. 478488.

[10] Egenfelt-Nielson. (2005). Beyond Edutainment: Exploring the Educational Potential of Computer Games.

[11] Gee, J.P. (2003). What Video Games Have to Teach us about Learning and Literacy. New York, NY. Palgrave Macmillan.

[12] Oppenheimer, T. (2003). A Flickering Mind: The False Promise of Technology in the Classroom. New York: Random House. 